\section{Fatores associados à prática de exercícios físicos em homens voluntários adultos e idosos residentes na Grande São Paulo, Brasil}

\section{Factors associated to physical exercise in adults and elderly volunteer men living in S. Paulo, Brazil}

\author{
Alex Antonio Florindo \\ Departamento de Epidemiologia \\ Faculdade de Saúde Pública \\ Universidade de São Paulo \\ Endereço para correspondência: \\ Rua Euclides Coelho, 101 \\ 03919-060 São Paulo, SP - Brasil \\ E-mail: aflorind@usp.br
}

\section{Maria do Rosário Dias de Oliveira Latorre \\ Departamento de Epidemiologia \\ da Faculdade de Saúde Pública \\ Universidade de São Paulo}

Tomoe Tanaka

Departamento de Epidemiologia

Faculdade de Saúde Pública

Universidade de São Paulo

\section{Patrícia Constante Jaime}

Departamento de Epidemiologia

Faculdade de Saúde Pública

Universidade de São Paulo

\section{Cristiano Augusto de Freitas Zerbini}

Hospital Heliópolis, São Paulo - SP

\section{Resumo}

O objetivo deste estudo foi verificar os fatores associados à prática de exercícios físicos em homens adultos e idosos residentes na grande São Paulo. O estudo foi transversal e a população constou de 326 homens voluntários, avaliados no Hospital Heliópolis em São Paulo. As variáveis de estudo foram a prática de exercícios físicos (PEF) dos últimos 12 meses (variável dependente), e idade, nível de escolaridade, prática de exercícios físicos no período de 10 a 30 anos, cor da pele, estado civil, tabagismo, ingestão de bebidas alcoólicas, ocupação e índice de massa corporal foram as variáveis independentes. A análise univariada foi realizada através do teste de $\chi^{2}$ e regressão logística simples e a análise conjunta através de regressão logística múltipla. A variável que melhor explicou a PEF foi o nível de escolaridade. Depois do ajuste por tabagismo, idade e IMC, os homens com ensino fundamental e médio completos mostraram maior chance de PEF (OR=4,56; $\mathrm{IC}_{95} \%=1,66-12,50$ ), o mesmo acontecendo com homens com ensino superior $\left(\mathrm{OR}=8,39 ; \mathrm{IC}_{95} \%=1,83-38,42\right)$, quando comparados com homens com escolaridade até ensino fundamental incompleto. Estes resultados são importantes para as políticas públicas de implantação e implementação de programas de exercícios físicos, mostrando ser de fundamental importância programas que objetivem atingir a população masculina de adultos e idosos com baixa escolaridade na grande São Paulo.

Palavras-chave: Exercício Físico. Educação. Adulto. Idoso. Homens.

Trabalho realizado no Departamento de Epidemiologia da Faculdade de Saúde Pública da Universidade de São Paulo e no Hospital Heliópolis em São Paulo -SP. 


\section{Abstract}

The aim of the study was to analyze the factors associated to practicing physical exercise in adults and elderly men living in S. Paulo. It is a cross-sectional study that analyzes 326 volunteer men aged 50 years and older. The dependent variable was the practice of physical exercise (PPE) in the last 12 months and the independent variables were age, education, practice of physical exercise in the period from 10 to 30 years of age, race, marital status, smoking habits, ingestion of alcoholic drinks, occupation and body mass index (BMI). The analysis was done using the $\chi^{2}$ test and simple logistic regression and multiple logistic regression. Education was associated to PPE, even after adjusting for smoking habits, age and BMI. Men with elementary school $(\mathrm{OR}=4.56$, $95 \mathrm{CI} \%=1.66-12.50)$ and men with college education $(\mathrm{OR}=8.39,95 \mathrm{CI} \%=1.83-38.42)$ practice more physical exercise than men that did not finish elementary school. These results show that education should be considered in the planning of physical exercise programs for adults and elderly men.

Keywords: Physical exercise. Education. Adult. Aged. Men.

\section{Introdução}

As atividades físicas e os exercícios físicos são considerados importantes estratégias de promoção em saúde e prevenção de doenças ${ }^{1,2}$. O sedentarismo é um problema de saúde pública em algumas regiões do Brasil, principalmente nas grandes capitais ${ }^{3} \mathrm{e}$ no município de São Paulo ${ }^{4,5}$, em virtude da baixa prevalência de pessoas praticantes de exercícios físicos nestas regiões. Acredita-se que este fato seja decorrente de fatores estruturais que influenciam a adesão da população à prática de exercícios físicos $(\mathrm{PEF})^{6}$.

Já é conhecido, em países desenvolvidos, que a maior adesão à PEF está associada com maiores níveis de escolaridade e de renda ${ }^{7-}$ ${ }^{11}$. Porém, estes fatores são pouco estudados em países em desenvolvimento como o Brasil. Visto que a adesão a PEF é de extrema importância como parte do conjunto de programas direcionados à promoção em saúde, o objetivo do presente estudo foi verificar os fatores associados à prática de exercícios físicos em homens adultos e idosos residentes na grande São Paulo, utilizando os dados da pesquisa "Determinantes da Densidade Mineral Óssea em Homens Adultos e Idosos"12.

\section{Material e Métodos}

Este foi um estudo do tipo transversal. A população constou de 326 homens com idade igual ou superior a 50 anos que foram convidados para participar da pesquisa "Determinantes da Densidade Mineral Óssea em Homens Adultos e Idosos", realizada no Hospital Heliópolis, localizado na região sul do município de São Paulo. A área de abrangência do Hospital inclui a região Sul e municípios vizinhos como São Caetano do Sul, São Bernardo do Campo, Santo André e Diadema.

A divulgação da pesquisa foi realizada através de anúncio em jornal do bairro do Ipiranga e adjacências, convites para homens acompanhantes de mulheres em consulta periódica no Posto de Atendimento Médico do Hospital e convites para funcionários do 
Hospital através de divulgação interna. Todos os participantes foram convidados especificamente para realizar a análise da densidade mineral óssea.

Para investigação da atividade física habitual, utilizou-se o questionário de Baecke et al. ${ }^{13}$. Este questionário compõe-se de 16 questões referentes às atividades físicas ocupacionais, exercícios físicos, atividades físicas de lazer e atividades físicas de locomoção. O questionário foi traduzido e adaptado para língua portuguesa com autorização dos autores ${ }^{12}$. Para o presente estudo, utilizaram-se as informações referentes a PEF dos últimos 12 meses e também a prática de exercícios físicos referente ao período de 10 a 30 anos de idade.

Para a investigação das variáveis sóciodemográficas, utilizou-se questionário. Foram coletados dados de escolaridade, cor da pele, tabagismo, estado civil, ingestão de bebidas alcoólicas, idade, naturalidade, utilização de medicamentos e antecedentes mórbidos. Todos os dados referentes ao questionário de atividade física habitual e questionário de variáveis sócio-demográficas foram coletados através de entrevista individual por uma técnica especificamente treinada com supervisão dos pesquisadores. Foram coletados dados de antropometria (peso e estatura) por uma única nutricionista e calculado o índice de massa corporal (IMC).

Para análise de dados, considerou-se como variável dependente a $\mathrm{PEF}$ dos últimos 12 meses (pessoas praticantes com $y=1$ e não praticantes com $y=0$ ). As demais variáveis (idade, nível de escolaridade, prática de exercícios físicos no período de 10 a 30 anos, cor da pele, estado civil, tabagismo, ingestão de bebidas alcoólicas, ocupação e IMC) foram consideradas como variáveis independentes. Com relação à cor da pele, resolveu-se juntar os amarelos com os pretos e pardos, em virtude da pequena quantidade de pessoas nesta categoria. No IMC, os indivíduos foram classificados de acordo com a padronização da Organização Mundial da Saúde ${ }^{14}$ (desnutrição para IMC <18,5; normal para IMC de 18,5 a 24,99; sobrepeso para IMC de 25,0 a 29,99; obesidade para IMC $>29,99$ ). Nesta classificação, optou-se por colocar os desnutridos junto com os normais, em virtude da pequena quantidade de pessoas desnutridas e para diferenciar dos homens com sobrepeso e obesidade. No estado civil, optou-se por agrupar os solteiros, separados, desquitados e viúvos (sem companheiras) para diferenciar dos casados (com companheiras). Na idade, optouse por dividir os homens em adultos (de 50 a 60 anos) e idosos (acima de 60 anos). Na ocupação, analisaram-se os aposentados separados dos trabalhadores na ativa. Na escolaridade, optou-se por dividi-la em três níveis, sendo o primeiro até o ensino fundamental incompleto, o segundo do ensino fundamental completo até o ensino médio completo e o terceiro, o ensino superior.

Numa primeira etapa foi realizada análise descritiva. A seguir, foi feita a análise univariada utilizando-se o teste de $\chi^{2}$ e a regressão logística não condicional. Para a elaboração do modelo final de regressão logística múltipla, foram selecionadas as variáveis independentes com $\mathrm{p}<0,20$ na análise univariada. A variável que permaneceu no modelo final foi estatisticamente significativa ou foi considerada como variável de controle. Utilizaram-se os softwares Epi-Info 6.04 e Stata 6.0.

O estudo foi aprovado pelo Comitê de Ética em Pesquisa da Faculdade de Saúde Pública da Universidade de São Paulo e pelo Comitê de Ética do Hospital Heliópolis, e todos os participantes assinaram o termo de consentimento livre e esclarecido da pesquisa.

\section{Resultados}

A proporção de PEF dos últimos 12 meses entre a população foi de $6 \%$, pois dos homens estudados, 306 (94\%) relataram não praticar nenhum tipo de exercício físico nos últimos 12 meses. Observou-se que os tipos de exercícios físicos mais praticados foram as modalidades de natação e ginástica geral (Figura). A média, em minutos por semana, de PEF praticada foi de 150 minutos ( $\mathrm{DP}=87,1$ minutos) e em média, a PEF foi realizada du- 


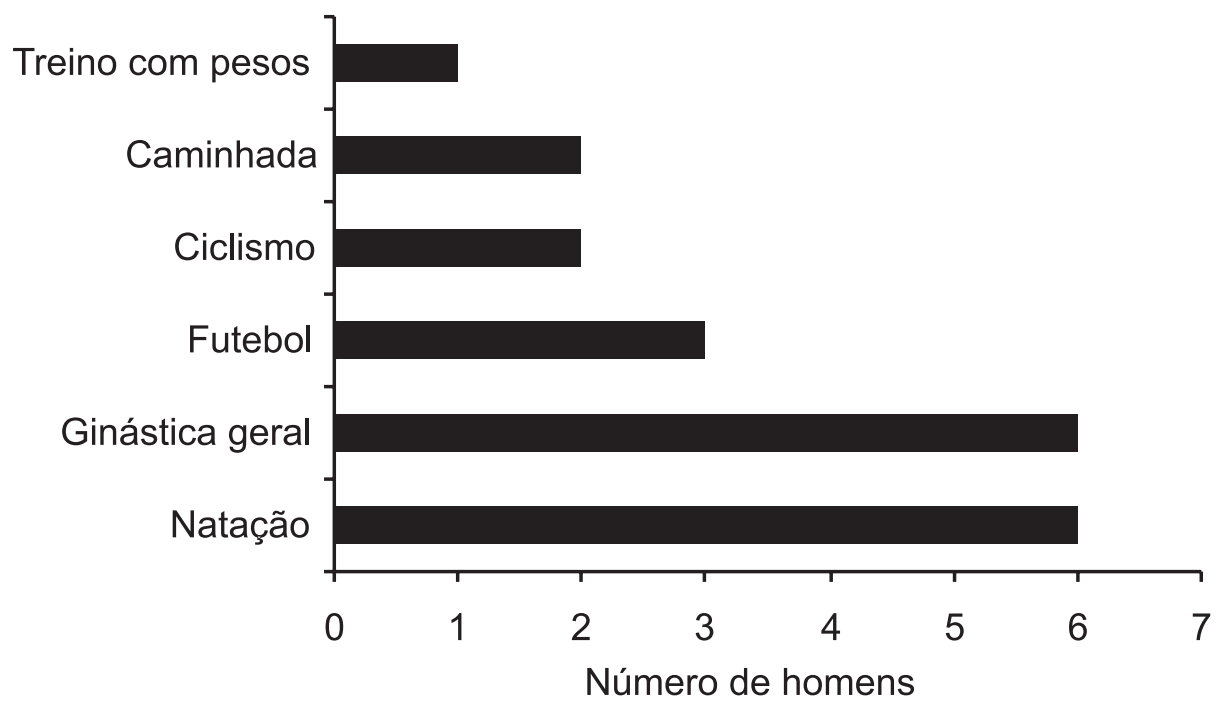

Figura - Modalidades de PEF praticadas por homens adultos e idosos da região da grande São Paulo, Hospital Heliópolis, 1997.

Figure - Modalities of PPE practised for adult and elderly men S.Paulo, Heliopolis Hospital, 1997.

rante nove meses no ano $(\mathrm{DP}=2,7$ meses $) . \mathrm{E}$ interessante ressaltar que apenas cinco homens $(1,5 \%)$ relataram praticar uma segunda modalidade de exercício físico, sendo que, destas modalidades, a mais praticada foi o futebol (dois homens), seguido por treinamento com pesos (um homem), caminhada (um homem) e corrida (um homem).

A média de idade da população de estudo foi de 62,5 anos (DP=7,9 anos), sendo a maioria com cor de pele branca $(80 \%)$, casada (87\%) e com nível de escolaridade até o ensino fundamental incompleto (74\%). Com relação às medidas antropométricas, a média de peso foi de 73,1 Kg ( $\mathrm{DP}=12,8 \mathrm{Kg})$, a média de estatura foi de 165,3 cm (DP=6,6 $\mathrm{cm})$ e a média do IMC foi de $26,7 \mathrm{Kg} / \mathrm{m}^{2}$ $\left(\mathrm{DP}=4,1 \mathrm{Kg} / \mathrm{m}^{2}\right)$. A maioria dos homens foi classificada com sobrepeso (42\%) ou com algum grau de obesidade (22\%) de acordo com a classificação da Organização Mundial da Saúde ${ }^{14}$.

A Tabela 1 apresenta os resultados de associação de cada variável independente com a variável dependente PEF e os respectivos valores de odds ratio (OR) bruto. Observa-se que a única variável estatisticamente significativa foi o nível de escolaridade
( $p=0,003)$ e decidiu-se avaliar o efeito da escolaridade, ajustada por idade, tabagismo e IMC, através da análise múltipla.

A Tabela 2 apresenta os OR's de escolaridade ajustados por idade, tabagismo e IMC. Verifica-se que esta variável esteve associada significativamente com a PEF dos últimos 12 meses, mesmo após a inclusão das outras variáveis no modelo. Quando ajustado pelas variáveis tabagismo, idade e IMC, os homens com ensino fundamental e ensino médio completos possuíram 4,56 vezes a chance de PEF, nos últimos 12 meses, em relação aos homens com ensino fundamental incompleto. Para os homens com nível superior completo a chance subiu para 8,39 vezes.

\section{Discussão}

Este foi um estudo transversal para avaliar quais características sócio-demográficas poderiam influenciar na PEF em homens adultos e idosos.

A população de estudo não é amostra representativa da população brasileira, pois foi composta por homens voluntários, porém, considera-se que não houve viés de 
Tabela 1 - Distribuição dos participantes da pesquisa segundo as variáveis de estudo, Hospital Heliópolis, São Paulo, 1997 Table 1 - Distribution of the participants of according to variables of study, Heliopolis Hospital, S.Paulo, 1997

\begin{tabular}{|c|c|c|c|c|c|c|c|c|}
\hline \multirow[t]{3}{*}{ Variável } & \multirow[t]{3}{*}{ Categoria } & \multicolumn{4}{|c|}{ Exercício físico } & \multirow[t]{3}{*}{ Total \% } & \multirow[t]{3}{*}{ OR bruto } & \multirow[t]{3}{*}{$\mathrm{p}^{*}$} \\
\hline & & \multicolumn{2}{|c|}{ Sim } & \multicolumn{2}{|c|}{ Não } & & & \\
\hline & & $\mathrm{N}^{\circ}$ & $\%$ & $\mathrm{~N}^{\circ}$ & $\%$ & & & \\
\hline \multirow[t]{2}{*}{ Cordapele } & Branca & 18 & 7 & 243 & 93 & 100 & 2,33 & \\
\hline & Não branca & 2 & 6 & 63 & 94 & 100 & 1 & 0,308 \\
\hline \multirow{2}{*}{ PEF de 10 a 30 anos } & Praticou & 12 & 7 & 160 & 93 & 100 & 1,37 & \\
\hline & Não praticou & 8 & 5 & 146 & 95 & 100 & 1 & 0,503 \\
\hline \multirow[t]{2}{*}{ Tabagismo } & Fumante & 1 & 2 & 64 & 98 & 100 & 5,02 & \\
\hline & Não fumante & 19 & 7 & 242 & 93 & 100 & 1 & 0,084 \\
\hline \multirow[t]{2}{*}{ Consumo de álcool } & Não & 13 & 5 & 230 & 95 & 100 & 0,61 & \\
\hline & Sim & 7 & 8 & 76 & 92 & 100 & 1 & 0,312 \\
\hline \multirow[t]{3}{*}{ Estado civil } & Casado & 19 & 7 & 264 & 93 & 100 & 3,02 & \\
\hline & Solteiro, viúvo, desquitado & & & & & & & \\
\hline & ou separado & 1 & 2 & 42 & 98 & 100 & 1 & 0,264 \\
\hline \multirow[t]{5}{*}{ Escolaridade } & Ensino superior & 3 & 20 & 12 & 80 & 100 & 7,25 & \\
\hline & $\begin{array}{l}\text { Ensino fundamental } \\
\text { completo até o ensino }\end{array}$ & & & & & & & \\
\hline & médio completo & 9 & 13 & 62 & 87 & 100 & 4,21 & \\
\hline & Ensino fundamental & & & & & & & \\
\hline & Incompleto & 8 & 3 & 232 & 97 & 100 & 1 & 0,003 \\
\hline \multirow[t]{2}{*}{ Idade } & 50 a 60 anos & 7 & 5 & 130 & 95 & 100 & 0,73 & \\
\hline & Acima de 60 anos & 13 & 7 & 176 & 93 & 100 & 1 & 0,511 \\
\hline \multirow[t]{2}{*}{$I M C$} & Normal e desnutrição & 6 & 5 & 107 & 95 & 100 & 0,75 & \\
\hline & Sobrepeso e obesidade & 14 & 7 & 199 & 93 & 100 & 1 & 0,571 \\
\hline \multirow[t]{3}{*}{ Ocupação atual } & Aposentado & 14 & 6 & 221 & 94 & 100 & 0,89 & \\
\hline & Trabalha & 6 & 7 & 85 & 93 & 100 & 1 & 0,830 \\
\hline & Total & 20 & & 306 & & 100 & & \\
\hline
\end{tabular}

*Nível descritivo de associação pelo teste de $\chi^{2}$

seleção, tendo em vista que eles se apresentaram para fazer exame de densitometria óssea e não avaliação da PEF.

Na comparação da população do presente estudo com dados da população brasileira, o levantamento de dados da pesquisa de padrão de vida $(\mathrm{PPV})^{3}$, realizado pelo Instituto Brasileiro de Geografia e Estatística (IBGE), mostrou que $57 \%$ da população masculina de todas as idades das grandes metrópoles do Brasil foram considerados como estando dentro do peso normal, contra $35 \%$ das pessoas deste estudo, o que mostra um percentual maior de obesos no presente estudo, provavelmente por se trabalhar com homens com idade igual ou superior a 50 anos. Analisando-se a Pesquisa Nacional sobre Saúde e Nutrição (PNSN) ${ }^{3}$, realizada pelo IBGE em 1989, verifica-se que aumentou a proporção de obesos, sendo que 
Tabela 2 - OR para prática de exercício físico ajustada pelas variáveis nível de escolaridade, tabagismo, idade e IMC.

Table 2 - OR for practical of physical exercise adjusted by education, smoking, age and BMI

\begin{tabular}{llccc}
\hline Variável & Categoria & OR bruto & OR ajustado & $\mathrm{IC}_{95 \%}$ (ORajust) \\
\hline Escolaridade & $\begin{array}{l}\text { Ensino superior } \\
\text { Ensino fundamental } \\
\text { completo até o ensino } \\
\text { médio completo }\end{array}$ & 7,25 & 8,38 & $1,83-38,40$ \\
& $\begin{array}{l}\text { Até o ensino fundamental } \\
\text { incompleto }\end{array}$ & 4,21 & 4,56 & $1,66-12,50$ \\
& & 1,0 & 1,0 & - \\
Tabagismo & Não fumante & 5,02 & 4,30 & $0,53-34,64$ \\
& Fumante & 1,0 & 1,0 & - \\
Idade & 50 a 60 anos & 0,73 & 0,60 & $0,22-1,67$ \\
& Acima de 60 anos & 1,0 & 1,0 & - \\
IMC & $\begin{array}{l}\text { Desnutridos e normais } \\
\text { Sobrepeso e obesos }\end{array}$ & 0,75 & 0,91 & $0,32-2,53$ \\
& & 1,0 & 1,0 & - \\
\hline
\end{tabular}

entre os homens, a proporção de obesos subiu de $4,5 \%$ na PNSN para $7,0 \%$ na PPV. Somando-se os homens com sobrepeso e obesidade (64\%) deste estudo observa-se uma tendência parecida com a população brasileira das grandes capitais.

Na análise dos níveis de escolaridade, de acordo com dados da Fundação Seade ${ }^{15}$ (1999), a taxa de analfabetismo para o município de São Paulo no ano de 1995 foi de $7,69 \%$. Sabe-se que o analfabetismo é mais prevalente na população com 50 anos ou mais. Dados da população do estado de São Paulo de 1996 indicam que $21,6 \%$ da população com 50 anos ou mais eram analfabetos ou tinham menos de um ano de estudo ${ }^{16}$. A população deste estudo apresentou $2,1 \%$ de analfabetos e $75 \%$ dos homens com escolaridade até o ensino fundamental incompleto. Apesar do baixo percentual médio de analfabetos em comparação com os dados gerais da população do município de São Paulo e da população com 50 anos ou mais do estado de São Paulo, de acordo com a Pesquisa de Condições de Vida ${ }^{17}$ realizada em 1998, observou-se que $73 \%$ da população do Estado de São Paulo possuíam até o ensino médio incompleto, muito semelhan- te a esta população de estudo. Portanto, considera-se que, embora ela não seja representativa da população da grande São Paulo, estes homens possuem características semelhantes, relativas à escolaridade.

Em estudos epidemiológicos, o método mais recomendado para a investigação da atividade física habitual é a avaliação através de questionários ${ }^{18,19}$. Especificamente com relação ao questionário utilizado neste estudo, observou-se que não houve dificuldade na avaliação das atividades físicas, principalmente a PEF. A estrutura das questões foi elaborada de modo a eliminar a influência que poderia sofrer quando aplicado a diferentes línguas e culturas. As questões de PEF foram caracterizadas por qual o tipo de exercício físico ou esporte praticado nos últimos doze meses, quantas horas por semana que praticou e quantos meses por ano que praticou nos últimos doze meses. Portanto, acredita-se que não houve problemas na aplicação destas questões nesta população.

Já é conhecida a relação inversa entre níveis moderados a vigorosos de PEF, com níveis de morbidade e mortalidade por doenças cardiovasculares e problemas como a obesidade $^{1,2,20}$, principalmente em pessoas 
com idade igual ou superior a 50 anos. A $\mathrm{PPV}^{3}$ indicou que $30 \%$ da população brasileira residente nas grandes capitais está engajada em algum tipo de PEF. Estudo epidemiológico recente no município de São Pau$10^{4}$ mostrou que de 1.000 indivíduos estudados, $30 \%$ praticavam algum tipo de exercício físico. Nunomura ${ }^{21}$ cita um levantamento sobre atividades físicas realizado pelo Instituto Datafolha, em 98 municípios do Brasil com 2.054 pessoas entre 18 a 60 anos de idade no ano de 1997. Os resultados mostraram que $40 \%$ da população praticavam algum tipo de exercício físico, sendo que, dos que praticavam, $38 \%$ praticavam todos os dias, $62 \%$ possuíam curso superior, $57 \%$ tinham renda superior a 20 salários mínimos, $50 \%$ eram homens, $47 \%$ residiam na região sul e $46 \%$ tinham idade entre 18 a 24 anos. Dos que não praticavam, $70 \%$ eram mulheres, $69 \%$ tinham escolaridade até o ensino fundamental, $66 \%$ tinham idade entre 45 e 60 anos, $65 \%$ residiam na região Nordeste e $64 \%$ tinham renda até dez salários mínimos. Pesquisa epidemiológica realizada em mulheres e homens adultos e idosos australianos ${ }^{22}$ mostrou uma proporção inversa, com um índice de PEF em $70 \%$ da população contra $30 \%$ de não praticantes. No presente estudo, $94 \%$ não praticavam nenhum tipo de exercício físico. Acredita-se que esta baixa proporção de PEF deve-se principalmente a faixa etária estudada, pois quanto maior a idade, menor a proporção de PEF como apontam as pesquisas em mulheres e homens norte-americanos ${ }^{23} \mathrm{e}$ australianos ${ }^{22}$.

$\mathrm{O}$ estudo de Nunomura ${ }^{21}$ mostrou que os principais motivos de adesão a PEF estão relacionados a realização dos exercícios físicos em grupos, ao condicionamento físico, a saúde e a forma física ou estética respectivamente. Voltando a discutir o levantamento realizado pelo Datafolha ${ }^{21}$, os cinco principais motivos alegados para não se exercitar foram a falta de tempo (65\%), não gostar de esporte (18\%), ter preguiça ( $10 \%)$, falta de dinheiro (10\%) e problemas de saúde (7\%), respectivamente. Os principais motivos alegados para prática foram emagrecimento ou manutenção da forma física (53\%), manu- tenção ou melhora da saúde (36\%), hábito de vida (20\%), recomendação médica (16\%) e lazer (11\%). Porém, em países como o Brasil, existem outros problemas estruturais ${ }^{6}$ como a escassez e precariedade de instalações para a PEF, baixo nível socioeconômico da população e ausência de tempo ${ }^{24}$, os quais também são fatores associados a baixa proporção de PEF. Estudos de países desenvolvidos têm indicado que maiores níveis de renda são importantes determinantes da PEF em mulheres e homens adultos norte-americanos ${ }^{23} \mathrm{e}$ australianos ${ }^{22}$, em idosos holandeses $^{7}$, em adultos e idosos canadenses ${ }^{25} \mathrm{e}$ dinamarqueses ${ }^{26}$. É interessante ressaltar os resultados do estudo de Monteiro et al. ${ }^{27}$ realizado em policiais militares da cidade de Bauru no estado de São Paulo. Os homens com maior nível socioeconômico, incluindo renda per capita maior que dois salários mínimos, casa própria, sem dependentes e sem uma segunda ocupação, estavam engajados em PEF regular e eram mais ativos que os homens com renda per capita até um salário mínimo, que não possuíam casa própria, que possuíam dependentes e que tinham maiores cargas de trabalho.

Com relação aos níveis de escolaridade, no município de São Paulo, a menor prevalência de PEF está concentrada nas classes sociais D e E, caracterizadas por classes com menores ganhos familiares, menor poder de compra e baixos níveis de escolaridade ${ }^{4}$.

No presente estudo, a única variável que explicou a PEF dos últimos 12 meses em homens adultos e idosos foi a escolaridade, mesmo depois de ajustada por tabagismo, idade e IMC.

Estes resultados são semelhantes às pesquisas realizadas em mulheres e homens adultos de países desenvolvidos como Austrália ${ }^{22}$, Inglaterra, Escócia e País de Gales ${ }^{8,9}$, Estados Unidos ${ }^{10}$, Canadá ${ }^{25}$ e Dinamarca ${ }^{26}$, que mostraram que maiores níveis de escolaridade são importantes determinantes para adesão a PEF e para maiores níveis de atividades físicas.

O tabagismo também está relacionado com baixa adesão a PEF e com maiores níveis de inatividade física em mulheres e ho- 
mens norte-americanos ${ }^{23,26,28}$. Estudos em mulheres e homens adultos norte-america$\operatorname{nos}^{23}$ e australianos ${ }^{22}$, e em homens idosos holandeses ${ }^{7}$, mostraram que o nível de atividades físicas está inversamente correlacionado com a idade. Além disso, alguns trabalhos mostraram que maiores níveis de IMC estão associados com menores níveis de atividades físicas e de adesão a PEF em mulheres e homens norte-americanos ${ }^{23,10}$. Neste estudo não foi encontrada associação entre PEF e as variáveis tabagismo, idade e IMC. Argumenta-se que a associação não foi observada provavelmente pelo pequeno número de homens que relataram PEF. Porém, optou-se por deixar estas variáveis no modelo pela importância que representam na associação com a $\mathrm{PEF}^{7,10,22,23,26,28}$.

Considerando que o nível de escolaridade é um fator determinante para a PEF, seja em homens adultos e idosos da região da grande São Paulo, como mostrou este estudo, ou seja em populações de países desenvolvidos, como mostraram outras pesquisas $^{7-11}$, é importante que a implantação e implementação de programas de exercícios físicos tenha enfoque maior nas pessoas com baixo nível de escolaridade, para poder contribuir para o aumento na adesão à PEF. Recomenda-se, também, priorizar questões relacionadas à infra-estrutura, caracterizadas pelo aumento do número de clubes e parques públicos, além da melhoria dos já existentes, principalmente para a população de baixa renda, pois acredita-se que este fator também poderia contribuir para aumentar a adesão a PEF de homens adultos e idosos residentes na grande São Paulo.

\section{Agradecimentos}

Agradecemos à Fundação de Amparo a Pesquisa do Estado de São Paulo (FAPESP) pelas bolsas de mestrado e doutorado concedidas a Alex Antonio Florindo; ao Conselho Nacional de Pesquisas (CNPq) pela bolsa de pesquisa concedida a Maria do Rosário Dias de Oliveira Latorre, e pela bolsa de doutorado concedida a Tomoe Tanaka; à Coordenadoria de Aprimoramento Pessoal (CAPES) pela bolsa de doutorado concedida a Patrícia Constante Jaime.

\section{Referências}

1. Pate RR, Pratt M, Blair SN, Haskell WL, Macera CA, Bouchard C et al. Physical activity and public health: a recommendation from the centers for disease control and prevention and the american college of sports medicine. JAMA 1995; 273:402-7.

2. Blair SN, Kohl HW, Paffenbarger Jr. RS, Clark DG, Cooper KH, Gibbons LW. Physical fitness and allcauses mortality: a prospective study of health men and women. JAMA 1989; 262:2395-401.

3. Fundação IBGE. Pesquisa de Padrão de Vida [release on-line da pesquisa]. Disponível em <URL:http:// www.ibge.gov.br>. [1998 set 14].

4. Mello MT, Fernandez AC, Tufik S. Levantamento epidemiológico da prática de atividade física na cidade de São Paulo. Rev Bras Med Esport 2000; 6:119-24.
5. Rego RA, Berardo FAN, Rodrigues SSR, Oliveira ZMA, Oliveira MB, Vasconcellos $\mathrm{C}$ et al. Fatores de risco para doenças crônicas não transmissíveis: inquérito domiciliar no município de São Paulo, SP (Brasil). Metodologia e resultados preliminares. Rev Saúde Pública 1988; 24:277-85.

6. Florindo AA. Educação física e promoção em saúde. Rev Bras Ativ Fís Saúde 1998; 3:84-9.

7. van den Hombergh CE, Schouten EG, van Staveren WA, van Amelsvoort LG, Kok FJ. Physical activities of noninstitutionalized Dutch elderly and characteristics of inactive elderly. Med Sci Sports Exec 1995; 27:334-9. 
8. Eaton CB, Reynes J, Assaf AR, Feldman H, Lasater T, Carleton RA. Predicting physical activity change in men and women in two New England communities. Am J Prev Med 1993; 9:209-19.

9. Kuh DJL, Cooper C. Physical activity at 36 years: patterns and childhood predictors in a longitudinal study. J Epidemiol Community Health 1992; 46:14-9.

10. Schimtz K, French SA, Jeffery RW. Correlates of changes leisure time physical activity over 2 years: the Healthy Worker Project. Prev Med 1997; 26:570-9.

11. Bild DE, Jacobs DR Jr, Sidney S, Haskell WI, Andersen N, Oberman A. Physical activity in young black an white women. The CARDIA Study. Ann Epidemiol 1993; 3:636-44.

12. Florindo AA. Atividade física habitual e densidade mineral óssea em homens adultos e idosos [Dissertação de Mestrado]. São Paulo: Faculdade de Saúde Pública da USP; 2000.

13. Baecke JA, Burema J, Frijters JE. A short questionnaire for the measurement of habitual physical activity in epidemiological studies. Am J Clin Nutr 1982; 36:93642 .

14. World Health Organization. Physical status: the use and interpretation of antropometry. report of a World Health Organization study group. Geneva; 1995.

15. Fundação SEADE. Informações dos municípios paulistas [on line] . Disponível em <URL: http:// www.seade.gov.br/cgi-bin/titabpv98>. [1999 nov 06].

16. Instituto Nacional de Estudos e Pesquisas Educacionais - INEP 2000. Diagnóstico da situação educacional de jovens e adultos [on line]. Disponível em <URL: http://www.inep.gov.br>. [2001 jun 12].

17. Fundação SEADE. Pesquisa de Condições de Vida PCV 1998 [on line]. Disponível em <URL: http:// www.seade.gov.br> [1999 nov 06].

18. Nahas MV. Revisão de métodos para determinação dos níveis de atividade física habitual em diversos grupos populacionais. Rev Bras Ativ Fís Saúde 1996; 1:27-37.
19. Laporte RE, Montoye HJ, Caspersen CJ. Assessment of physical activity in epidemiologic research: problems and prospects. Public Health Rep 1985; 100:131-46.

20. Haapanen N, Miilunpalo S, Vuori I, Oja P, Pasanen M. Characteristics of leisure time physical activity associated with decreased risk of premature all-cause and cardiovascular disease mortality in middle-aged men. Am J Epidemiol 1996;143:870-80.

21. Nunomura M. Motivos de adesão à atividade física em função das variáveis idade, sexo, grau de instrução e tempo de permanência. Rev Bras Ativ Fís Saúde 1998; $3: 45-58$

22. Owen N, Bauman A. The decritive epidemiology of sedentary life-style in adult Australians. Int $\mathrm{J}$ Epidemiol 1992; 21:305-10.

23. Dietz WH. The role of lifestyle in health: the epidemiology and consequences of inactivity. Proc Nutr Soc 1996; 55:829-40.

24. Andrade DR, Matsudo SMM, Matsudo VKR, Figueira Jr. AJ, Araújo TL, Andrade EL et al. Barriers to exercise adherence among active young adults. In: $45^{\text {th }}$ Annual Meeting of American College of Sports Medicine; 1998 June 3-6; Orlando (USA). Med Sci Sports Exerc; 1998; 30 Suppl : S182.

25. Choiniere R, Lafontaine P, Edwards AC. Distribuition of cardiovascular disease risk factors by socioeconomic status among Canadian adults. CMAJ 2000; 162: S1324 .

26. Harreby M, Hesselsoe G, Kjer J, Neergaard K. Low back pain and physical exercise in leisure time in 38-yearold men and women: a 25-year prospective cohort study of 640 school children. Eur Spine J 1997; 6:181-6.

27. Monteiro HL, Gonçalves A, Padovani CR, Neto JLF. Fatores socioeconômicos e ocupacionais e a prática de atividade física regular: estudo a partir de policiais militares em Bauru, São Paulo. Motriz 1998; 4:91-7.

28. Dannenberg AL, Keller JB, Wilson PW, Castelli WP. Leisure time physical activity in the Framingham offspring study. Description, seasonal variation, and risk factor correlates. Am J Epidemiol 1989; 129: 76-88. 